\title{
Observational constraints on varying neutrino-mass cosmology
}

\author{
Chao-Qiang Geng, ${ }^{1,2,3, *}$ Chung-Chi Lee, ${ }^{2, \dagger}$ R. Myrzakulov, ${ }^{4, \ddagger}$ \\ M. Sami,${ }^{5, \S}$ and Emmanuel N. Saridakis ${ }^{6,7}$, ฯ \\ ${ }^{1}$ Chongqing University of Posts 85 Telecommunications, Chongqing, 400065, China \\ ${ }^{2}$ National Center for Theoretical Sciences, Hsinchu, Taiwan 300 \\ ${ }^{3}$ Department of Physics, National Tsing Hua University, Hsinchu, Taiwan 300 \\ 4 Eurasian International Center for Theoretical Physics, \\ Eurasian National University, Astana 010008, Kazakhstan \\ ${ }^{5}$ Centre for Theoretical Physics, Jamia Millia Islamia, New Delhi-110025, India \\ ${ }^{6}$ Physics Division, National Technical University of Athens, \\ 15780 Zografou Campus, Athens, Greece \\ ${ }^{7}$ Instituto de Física, Pontificia Universidad de \\ Católica de Valparaíso, Casilla 4950, Valparaíso, Chile
}

\begin{abstract}
We consider generic models of quintessence and we investigate the influence of massive neutrino matter with field-dependent masses on the matter power spectrum. In case of minimally coupled neutrino matter, we examine the effect in tracker models with inverse power-law and double exponential potentials. We present detailed investigations for the scaling field with a steep exponential potential, non-minimally coupled to massive neutrino matter, and we derive constraints on field-dependent neutrino masses from the observational data.
\end{abstract}

\footnotetext{
*Electronic address: geng@phys.nthu.edu.tw

$\dagger$ Electronic address: g9522545@oz.nthu.edu.tw

${ }^{\ddagger}$ Electronic address: rmyrzakulov@gmail.com

$\S$ Electronic address: sami@iucaa.ernet.in

『Electronic address: Emmanuel_Saridakis@baylor.edu
} 


\section{INTRODUCTION}

It is remarkable that both early and late time completions of the standard model of the universe include phases of accelerated expansion. Inflation [1-3] not only successfully addresses early time inconsistencies of the hot big bang, such as the flatness and horizon problems, but it also provides the mechanism of primordial perturbations needed for structure formation in the universe. On the other hand, the age crisis [4] in the standard cosmological model again asks for a late-time cosmic acceleration for its resolution in the standard lore. This phenomenon was directly confirmed by supernovae observations in 1998 [5, 6], and it was indirectly supported by other probes thereafter [7-9]. Such a confirmation for inflation is still awaited.

The late-time cosmic acceleration can be caused by the cosmological constant [10], with an energy scale of $O\left(10^{-3}\right) \mathrm{eV}$, or by a slowly-rolling scalar field (quintessence) with the mass of the order of $H_{0} \sim 10^{-33} \mathrm{eV}$ [11-14]. Both options are plagued with deep theoretical problems. Concerning the cosmological constant, it is an "unnatural" parameter [15] of Einstein theory, and its small numerical value at the classical level gets destabilized by quantum correction-vacuum energy. Concerning the fundamental scalar field, it faces the same problem of "naturalness". In Minkowski space time, vacuum energy can be safely ignored by choosing normal ordering in quantum field theory. In this case, it neither influences the dynamics nor there is a known way to measure it in local experiments. However, in curved space time, vacuum energy adds to the energy momentum of matter and it contributes to the dynamics of spacetime geometry. In the important case of Freidmann-Robertson-Walker (FRW) cosmology, geometry is conformally equivalent to Minkowski spacetime and thus one might naively think that it has solved the cosmological constant problem. In this case, however, one is left with a scalar field non-minimally coupled to matter in flat spacetime [16, 17]. Hence, the cosmological problem translates into an equivalent problem of naturalness of the scalar field.

Leaving the aforementioned problem aside, if we opt for quintessence then a specific behavior of the scalar-field dynamics is required, in order to keep the thermal history of the universe intact. Moreover, if quintessence has originated from inflation in the early universe in an attempt to attribute both accelerating phases in the same scalar field (quintessential inflation) [18-22], then the scaling behavior of the scalar field is required in order to comply 
with the nucleosynthesis constraint. The exit from the scaling regime to the late-time acceleration can be caused by invoking the non-minimal coupling with massive neutrino matter [23-25]. It is really mysterious that the neutrino mass is around the mass scale associated with dark energy. Once the non-minimal coupling to neutrino matter is invoked, the mass of the neutrino gets connected to the minimum of the effective potential and thereby to dark energy [22]. The neutrino mass modifies the cosmological evolution at both background and perturbation levels [26, 27]. Amongst others, the neutrino mass shifts the time of matter-radiation equality, and the free-streaming massive neutrino suppresses the growth of matter density perturbation within the free-streaming length scale [28, 29]. It is well known that particle-physics experiments give rise to constraints on neutrino masses. On the other hand, cosmology provides an independent probe, which is however sensitive to the new degree(s) of freedom over and above the standard model of particle physics. The massive neutrino matter crucially affects the matter power spectrum, and thus it can give rise to constraints on neutrino masses. In particular, the matter-density power spectrum is damped on small scales by massive neutrinos.

Having in mind the above discussion, it is both interesting and necessary to investigate the observational constraints on varying neutrino-mass cosmology. Although observational constraints analysis on varying-mass neutrino cosmology has been performed in the past (see for instance [30-35]) the crucial new feature of our investigation is the incorporation of the non-minimal coupling. In particular, we shall investigate quintessence models based upon the tracker field with massive neutrino matter minimally as well as non-minimally coupled to the field. Moreover, another novel feature is the use of the recently released Planck and SDSS data. The plan of the manuscript is the following: In section II, we consider dark energy scenarios based upon scalar field models, with inverse power law and double exponential potentials with minimally coupled neutrino matter. Section III is devoted to the dynamics of a scaling field coupled to massive neutrino matter. In section IV we present our results on the observational constraints on the above models. Finally, we give our conclusions in section $\mathrm{V}$. 


\section{QUINTESSENCE MINIMALLY COUPLED TO MATTER}

The quintessence scenario is one of the main paradigms for the description of the latetime acceleration [36]. In this set up, along with cold dark matter and baryonic component, we shall also be interested in considering massive neutrino matter. As mentioned above, this class of models can give rise to quintessential inflation, such that the dark energy scale emerges naturally in the scenario.

Before proceeding to non-minimal coupling with neutrino matter, we first briefly consider standard quintessence with minimally coupled massive neutrino matter. In order to set the notations, let us consider the following standard action

$$
\mathcal{S}=\int d^{4} x \sqrt{-g}\left[-\frac{M_{\mathrm{Pl}}^{2}}{2} R+\frac{1}{2} \partial^{\mu} \phi \partial_{\mu} \phi+V(\phi)\right]+S_{m}+S_{r},
$$

where $S_{m, r}$ denotes the action of ordinary matter including non-relativistic and relativistic one. Additionally, in order to describe the background cosmological evolution, we consider the spatially flat FRW geometry, in which case the action (1) leads to the following evolution equations

$$
\begin{aligned}
& 3 H^{2} M_{\mathrm{Pl}}^{2}=\rho_{m}+\rho_{r}+\rho_{\phi}, \\
& \left(2 \dot{H}+3 H^{2}\right) M_{\mathrm{Pl}}^{2}=-\left(p_{m}+p_{r}+p_{\phi}\right), \\
& \ddot{\phi}+3 H \dot{\phi}+\frac{d V}{d \phi}=0,
\end{aligned}
$$

where $\rho_{\phi}$ and $p_{\phi}$ denote the energy density and pressure of the quintessence field. In this case as usual, the dark energy sector is attributed to $\phi$ and the dark-energy equation-of-state parameter reads as $w_{D E}=w_{\phi} \equiv p_{\phi} / \rho_{\phi}$.

As mentioned in the introduction, specific features of scalar field dynamics such as tracker solutions are of interest in cosmology. In this case, once the conditions at the present epoch are set properly, cosmic evolution is not sensitive to the initial conditions. In what follows, we shall try to alleviate the problem associated with the said choice, for model parameters attributed to tracker solutions.

To make the point, we shall consider tracker solutions in the models with inverse powerlaw and double-exponential type potentials, though the underlying features are common to any tracker model. Let us begin with the inverse power-law type potential [13, 37-39]

$$
V(\phi)=V_{0}\left(\frac{\phi}{M_{\mathrm{Pl}}}\right)^{-n}
$$


where $V_{0}$ is a mass dimension-four constant. In this case, the slope of the potential is given by $\lambda_{\phi}=-M_{\mathrm{Pl}} V^{\prime} / V=n / \phi$, which is large at early times and diminishes at late times when $\phi$ acquires large values. Consequently, the field might mimic the background (scaling solution) at early epochs and could exit to slow roll at late times, giving rise to de Sitter attractor $a$ la a tracker solution. As explained in the Appendix, it is difficult to achieve tracker in this case for generic values of model parameters. For instance, for $V_{0} \sim M_{p}$, one requires much larger value of $n$ (see Ref. [20] for details). It turns out that it is much easier to realize tracker in a model with the double exponential potential.

Let us consider the potential of the form [40-42]

$$
V(\phi)=V_{0}\left(e^{-c_{1} \frac{\phi}{M_{\mathrm{P} 1}}}+e^{c_{2} \frac{\phi}{M_{\mathrm{Pl}}}}\right)
$$

where $V_{0}, c_{1}$ and $c_{2}$ are constants. For $\phi_{i} \gg 0$ at the initial time $(N \equiv \ln a \rightarrow-\infty)$, the second term in (6) can give rise to scaling regime for generic values of $c_{2}>9$ consistent with nucleosynthesis constraints of $\Omega_{\phi}=3\left(1+w_{b}\right) / c_{2}^{2}<0.045$ [33, 43], where $w_{b}$ is the equationof-state of the background fluid, i.e., $w_{b}=1 / 3$ and 0 in radiation and matter dominated epochs, respectively. On the other hand, for late times, when $\phi$ approaches the origin, (6) exhibits a minimum with $V_{\text {min }} \sim V_{0}$ for $c_{2} \sim c_{1}$. The latter can suit our requirement if we choose $V_{0} \sim \rho_{c r}$.

In order to present the aforementioned behavior in a more transparent way, we numerically evolve the evolution equations and we depict the corresponding evolution in Fig. 1. Indeed, in the upper graph of Fig. 1, we depict the corresponding evolutions for the energy densities of radiation $\left(\rho_{r}\right)$, matter $\left(\rho_{m}\right)$ and quintessence field $\left(\rho_{\phi}\right)$, normalized to the matter energy density $\rho_{m}^{(0)}$ at present, as functions of $N \equiv \ln a$, for three choices of $V_{0}, c_{1}$ and $c_{2}$. In the lower graph of Fig. 1, we depict the corresponding evolution for the quintessence equation-of-state parameter $w_{\phi}$. Because the cosmic evolution is insensitive to the initial conditions in the exponential potential, we can choose initial values in a broad parameter space. In the calculation, $\phi / M_{\mathrm{Pl}}=30 / c_{2}$ and $\dot{\phi}=0$ are set at $N=-20$. As we observe, the density ratio $\rho_{\phi} / \rho_{m}^{(0)}$, depending on $c_{2}$ in the early universe, does not change in radiation and matter dominated eras. Finally, the behavior of $\phi$-field at early times, can be estimated by combining (4) and (6), leading to $w_{\phi}=w_{b}$. Hence, as the scale of $\rho_{\phi}$ is close to $V_{0}$, the quintessence field evolves into the $\Lambda$ CDM-like stage, where $w_{\phi} \rightarrow-1$. Note that because the late-time behavior of the double exponential potential is almost the same as that of the 

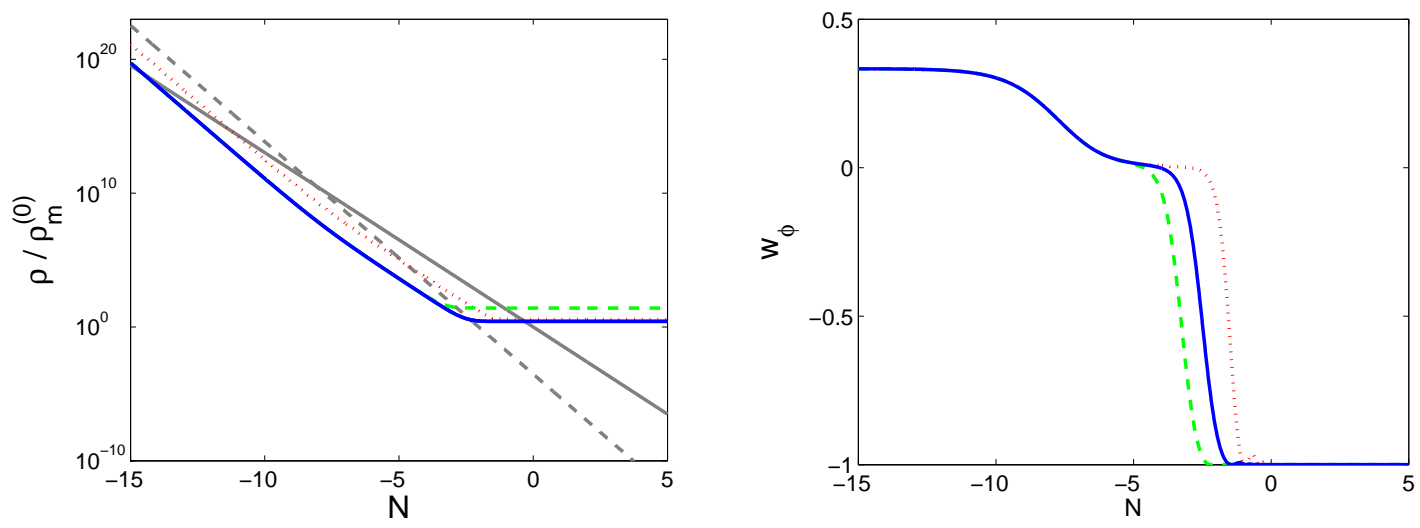

FIG. 1: Left: Evolutions of various energy densities, normalized to matter energy density $\rho_{m}^{(0)}$ at present $\left(a_{0}=1\right)$, as functions of $N \equiv \ln a$, for the minimally coupled scenario with the double-exponential potential $V=V_{0}\left(e^{-c_{1} \phi / M_{P l}}+e^{c_{2} \phi / M_{P l}}\right): \rho_{r}$ is the gray-dashed curve, $\rho_{m}$ is the gray-solid curve, and $\rho_{\phi}$ is plotted for three parameter choices, namely $\left(c_{1}, c_{2}, V_{0} / \rho_{m}^{(0)}\right)=(4,50,2)$ (blue-solid), $\left(c_{1}, c_{2}, V_{0} / \rho_{m}^{(0)}\right)=(4,50,20)$ (green-dashed) and $\left(c_{1}, c_{2}, V_{0} / \rho_{m}^{(0)}\right)=(4,10,2)$ (red-dotted). Right: The corresponding evolution of the quintessence equation-of-state parameter $w_{\phi}$. We have used $\rho_{r}^{(0)} / \rho_{m}^{(0)}=3 \times 10^{-4}$ as the boundary condition.

TABLE I: List of priors for parameters and allowed regions with 95\% C.L., and $V(\phi)=$ $V_{0}\left(e^{-c_{1} \phi / M_{\mathrm{Pl}}}+e^{c_{2} \phi / M_{\mathrm{P} 1}}\right)$ and $c_{1}=20$.

\begin{tabular}{lccc}
\hline \hline Parameter & Prior & Our result (95\% C.L.) & $\Lambda$ CDM $(95 \%$ C.L. $)$ \\
\hline Baryon density & $0.5<100 \Omega_{b} h^{2}<10$ & $100 \Omega_{b} h^{2}=2.20_{-0.05}^{+0.04}$ & $100 \Omega_{b} h^{2}=2.22_{-0.06}^{+0.04}$ \\
\hline CDM density & $10^{-3}<\Omega_{c} h^{2}<0.99$ & $\Omega_{c} h^{2}=0.118 \pm 0.003$ & $\Omega_{c} h^{2}=0.117_{-0.002}^{+0.004}$ \\
\hline Neutrino mass & $0<\Sigma m_{\nu}<1 \mathrm{eV}$ & $\Sigma m_{\nu}<0.200 \mathrm{eV}$ & $\Sigma m_{\nu}<0.198 \mathrm{eV}$ \\
\hline Spectral index & $0.9<n_{s}<1.1$ & $n_{s}=0.961 \pm 0.011$ & $n_{s}=0.963_{-0.011}^{+0.010}$ \\
\hline Potential & $10<c_{2}<40$ & $c_{2}>24.6$ & - \\
\hline
\end{tabular}

$\Lambda \mathrm{CDM}$ model, this scenario is hardly distinguished from $\Lambda$ CDM. We perform the CosmoMC package $[44,45]$ to extract the observational constraints and present the results of these two scenarios with one massive neutrino along with the other two being massless in Fig. 2 and Table. I. The obervational dataset will be introduced in Sec. IV.

It is clear from the above that the power-law potentials inevitably lead to a dark-energy 

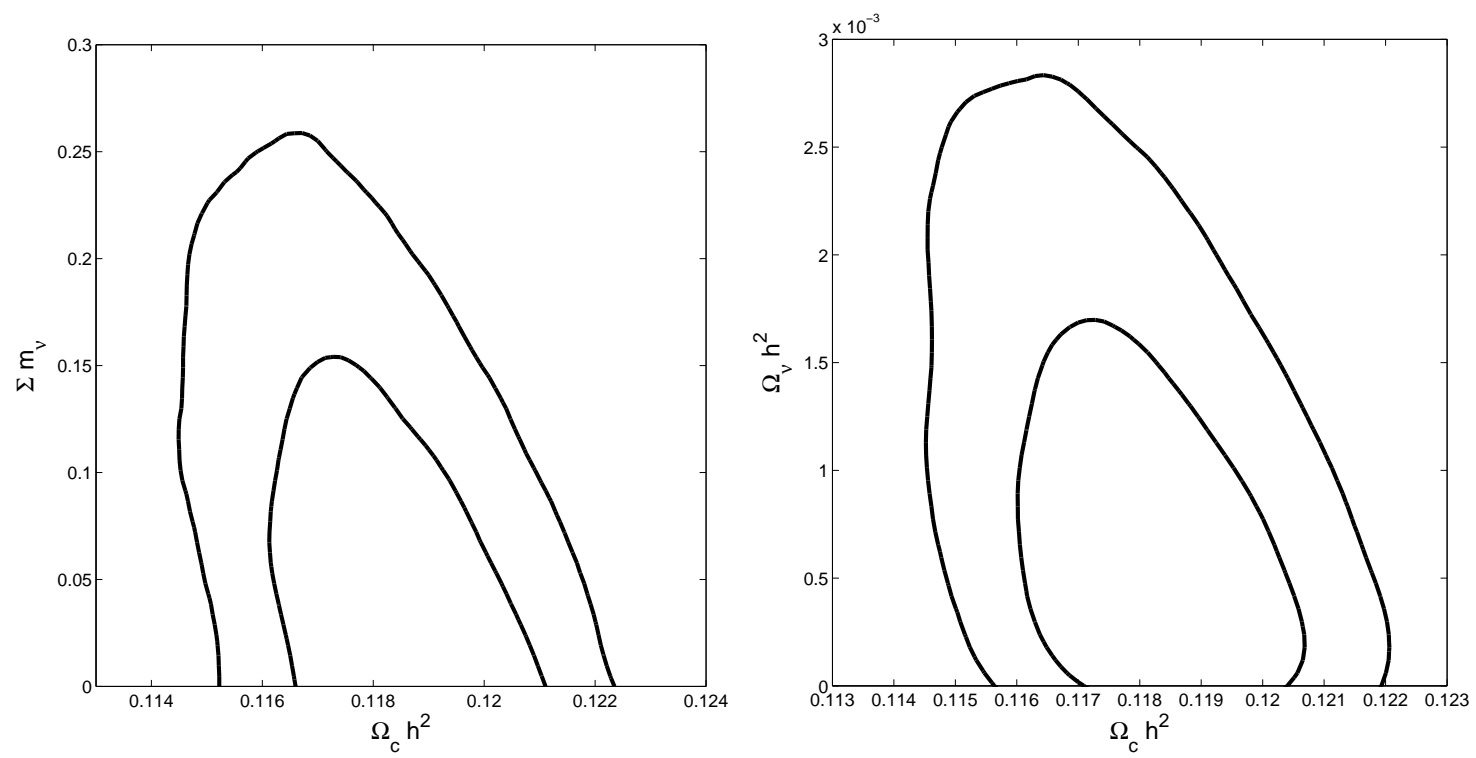

FIG. 2: Likelihood contours of the mass sum of the three neutrino species $\Sigma m_{\nu}$ and energy density ratio $\Omega_{\nu} h^{2}$ versus the CDM physical density $\Omega_{c} h^{2}$, for the minimally coupled scenario with the effective potential $V(\phi)=V_{0}\left(e^{-c_{1} \phi / M_{P l}}+e^{c_{2} \phi / M_{P l}}\right)$, where the inner and outer curves represent 1 and $2 \sigma$ confidence levels, respectively.

dominated epoch, and this $\phi$-dominated era occurs when $\rho_{\phi}$ is of the same order as $\rho_{m}$. However, for generic values of model parameters, the scenario is severely constrained by observations. On the other hand, the double exponential potential leads to a different behavior, in which a natural evolution in the radiation, matter and late-time acceleration regimes can be realized, provided that we choose appropriately the model parameters. In particular, the appearance of the dark energy dominated era crucially depends upon the scale of the potential $V_{0}$, which should be chosen around $\rho_{c r}$. The latter brings back the original problem associated with the cosmological constant.

In this section, we have performed the analysis in tracker models that have generic features related to the insensitivity of evolution to initial conditions. In this case, our results are close to the $\Lambda$ CDM paradigm [9]. This is not surprising since we are dealing with the slowlyrolling scalar field, which mimics the cosmological constant at late times. Therefore, we do not have significant improvements compared to the predictions of the $\Lambda$ CDM scenario. It is clear that a successful model of quintessence should maintain the scaling behavior, with an additional mechanism of exit to dark energy that could avoid the above disadvantage. This mechanism is provided by the non-minimal coupling between quintessence and massive 
neutrino matter. In the section to follow, we construct and analyze a model with the above features.

\section{NON-MINIMALLY COUPLED MASSIVE NEUTRINO MATTER AND GRACEFUL EXIT TO DARK ENERGY}

As mentioned above, one should look for a scenario in which the scale of dark energy emerges somewhat naturally. In this section we briefly review a model in which a key mechanism is the nonminimal coupling of the scalar field with varying-mass neutrinos [22]. In this case, $V_{0}$ gets linked to the energy density of massive neutrino matter.

The relevant action in the Einstein frame is written as

$$
\begin{aligned}
\mathcal{S}=\int & d^{4} x \sqrt{-g}\left[-\frac{M_{\mathrm{Pl}}^{2}}{2} R+\frac{1}{2} \partial^{\mu} \phi \partial_{\mu} \phi+V(\phi)\right] \\
& +\mathcal{S}_{\mathrm{m}}+\mathcal{S}_{\mathrm{r}}+\mathcal{S}_{\nu}\left(\mathcal{C}^{2} g_{\alpha \beta}, \Psi_{\nu}\right) .
\end{aligned}
$$

In the above expression, we have introduced a scalar field $\phi$ with $V(\phi)$ its potential, as well as matter and radiation sectors corresponding to perfect fluids. Note that due to the nonminimal curvature-neutrino coupling in the Jordan frame, we obtain a varying-mass neutrino sector in the Einstein frame, quantified by the coefficient [22]

$$
\mathcal{C}^{2}=A^{2} \mathrm{e}^{2 \beta \phi / M_{\mathrm{P} 1}}
$$

Hence, varying the action (7) with respect to the metric and specializing to the FRW case, we obtain the evolution equations

$$
\begin{aligned}
& 3 H^{2} M_{\mathrm{Pl}}^{2}=\frac{1}{2} \dot{\phi}^{2}+V(\phi)+\rho_{\mathrm{m}}+\rho_{\mathrm{r}}+\rho_{\nu}, \\
& \left(2 \dot{H}+3 H^{2}\right) M_{\mathrm{Pl}}^{2}=-\frac{1}{2} \dot{\phi}^{2}+V(\phi)-p_{m}-p_{\mathrm{r}}-p_{\nu},
\end{aligned}
$$

where $\rho_{i}$ are the energy densities of the corresponding sectors, and $p_{i}$ their pressures. Ad-

ditionally, varying the action (7) with respect to the scalar field, we derive its equation of motion, which reads

$$
\ddot{\phi}+3 H \dot{\phi}+\frac{\mathrm{d} V}{\mathrm{~d} \phi}=-\frac{\beta}{M_{\mathrm{Pl}}}\left(\rho_{\nu}-3 p_{\nu}\right),
$$

and thus the evolution equation for the neutrinos becomes

$$
\dot{\rho}_{\nu}+3 H\left(\rho_{\nu}+p_{\nu}\right)=\frac{\beta}{M_{\mathrm{Pl}}} \dot{\phi}\left(\rho_{\nu}-3 p_{\nu}\right) .
$$


Moreover, concerning radiation and matter sectors, we have the standard evolution. Additionally, for the neutrino sector we have

$$
m_{\nu, \mathrm{eff}}(\phi)=m_{\nu, 0} e^{\beta \phi / M_{\mathrm{Pl}}}
$$

and thus the neutrino pressure $p_{\nu}$ behaves as radiation during the early times, while it behaves as non-relativistic matter during late times [24] where non-minimal coupling builds up to induce non-zero neutrino masses. In this case, at late times, one obtains an effective dark energy sector, consisting of the scalar field as well as of the varying-mass neutrinos. In this case, the energy density and pressure of dark energy are given by

$$
\begin{aligned}
& \rho_{\mathrm{DE}} \equiv \rho_{\nu}+\rho_{\phi}=\rho_{\nu}+\frac{1}{2} \dot{\phi}^{2}+V(\phi), \\
& p_{\mathrm{DE}} \equiv p_{\nu}+p_{\phi}=p_{\nu}+\frac{1}{2} \dot{\phi}^{2}-V(\phi),
\end{aligned}
$$

which obey the standard continuity equation. Finally, as usual it proves to be convenient to introduce the dimensionless density parameters for radiation, matter, neutrinos and scalar field as

$$
\Omega_{i}=\frac{\rho_{i}}{3 H^{2} M_{\mathrm{Pl}}^{2}}, \quad(i=m, r, \nu, \phi)
$$

respectively, and therefore from (14) we obtain

$$
\Omega_{\mathrm{DE}}=\Omega_{\phi}+\Omega_{\nu}
$$

In order to obtain a viable model, we have to consider a specific ansatz for the potential $V(\phi)$ which is dictated by the requirement of nucleosynthesis. Hence, in order to illustrate the neutrino matter effect we consider the scaling potential, namely

$$
V(\phi)=V_{0} e^{-\alpha \phi / M_{\mathrm{P} 1}}
$$

The exponential potential in the non-minimally coupled quintessence model has been investigated with $\alpha<\sqrt{2}[32,35]$, satisfying the slow-roll potential condition. On the other hand, the scaling potential, $\alpha \gg 2$, has also been discussed to address the late-time acceleration occurring due to the effective potential of the non-minimally coupled neutrino matter [33]. Our work follows the later "naturalness" scaling potential with current observations. Thus, the scalar field equation of motion (11) becomes

$$
\ddot{\phi}+3 H \dot{\phi}+V_{e f f, \phi}=0
$$


where

$$
V_{e f f, \phi}=\frac{d V}{d \phi}+\frac{\beta}{M_{\mathrm{Pl}}}\left(\rho_{\nu}-3 p_{\nu}\right)
$$

is the derivative of the effective potential in which the scalar field moves, constituted of the original potential as well as of extra terms contributed by massive neutrino matter coupled to scalar field. The above cosmological scenario proves to exhibit a very interesting behavior, since this effective potential develops a minimum. We note that the latter provides an interesting mechanism for the unified description of inflation and late-time acceleration [24, 46]. Indeed, we easily check that the minimization of $V_{\text {eff }}$ requires,

$$
\phi_{\text {min }}=\frac{M_{\mathrm{Pl}}}{\alpha} \ln \left(\frac{V_{0}}{\gamma \rho_{\nu}}\right) ; \quad \beta \equiv \alpha \gamma
$$

which leads to

$$
V_{e f f}^{\min }=(1+\gamma) \rho_{\nu}\left(\phi_{\min }\right)
$$

where we have ignored the neutrino pressure at late times. We observe two features from (22). Firstly, it is clear that $V_{e f f}^{\min }$ is not very sensitive to $V_{0}$ and that the minimum of the effective potential is mainly defined by the numerical value of neutrino matter density at the present epoch. Secondly, the late-time dark energy density depends on the model parameters $\gamma$. It is interesting to note that $\omega_{D E} \equiv-1-2 \dot{H} / 3 H^{2}=-\gamma /(1+\gamma)$ at the present epoch. There is no much fine tuning in the numerical value of $\gamma$ and dark energy density in this case gets naturally connected to massive neutrino matter density at the present epoch. Note that we had introduced $\gamma$ for simplifying the analytical estimates though we do not need to use it any further. In our numerical elaboration we only consider $\alpha$ to be the free parameter, since it controls $\rho_{\phi} / \rho_{c}$ in matter and radiation dominated eras.

In order to examine the above behavior in more detail, we perform a numerical elaboration and we depict the corresponding evolution of the various energy densities, the quintessence equation-of-state parameter and the neutrino masses in Fig. 3. We should emphasize that thanks to the tracker behavior in the high redshift regime and the emergence of the darkenergy dominated era at late times, the scenario can successfully be embedded in the framework of quintessential inflation (see Ref. $[22,24]$ for details). Additionally, since the earlytime and late-time evolutions are insensitive to the initial conditions and $V_{0}$, respectively, we choose $\left(\phi / M_{\mathrm{Pl}}, \dot{\phi} / M_{\mathrm{Pl}}\right)=(-60 / \alpha, 0)$ at $N=-20$ and fix $V_{0}$ for convenience as $V_{0}=7 \rho_{m}^{(0)} / 3$, 

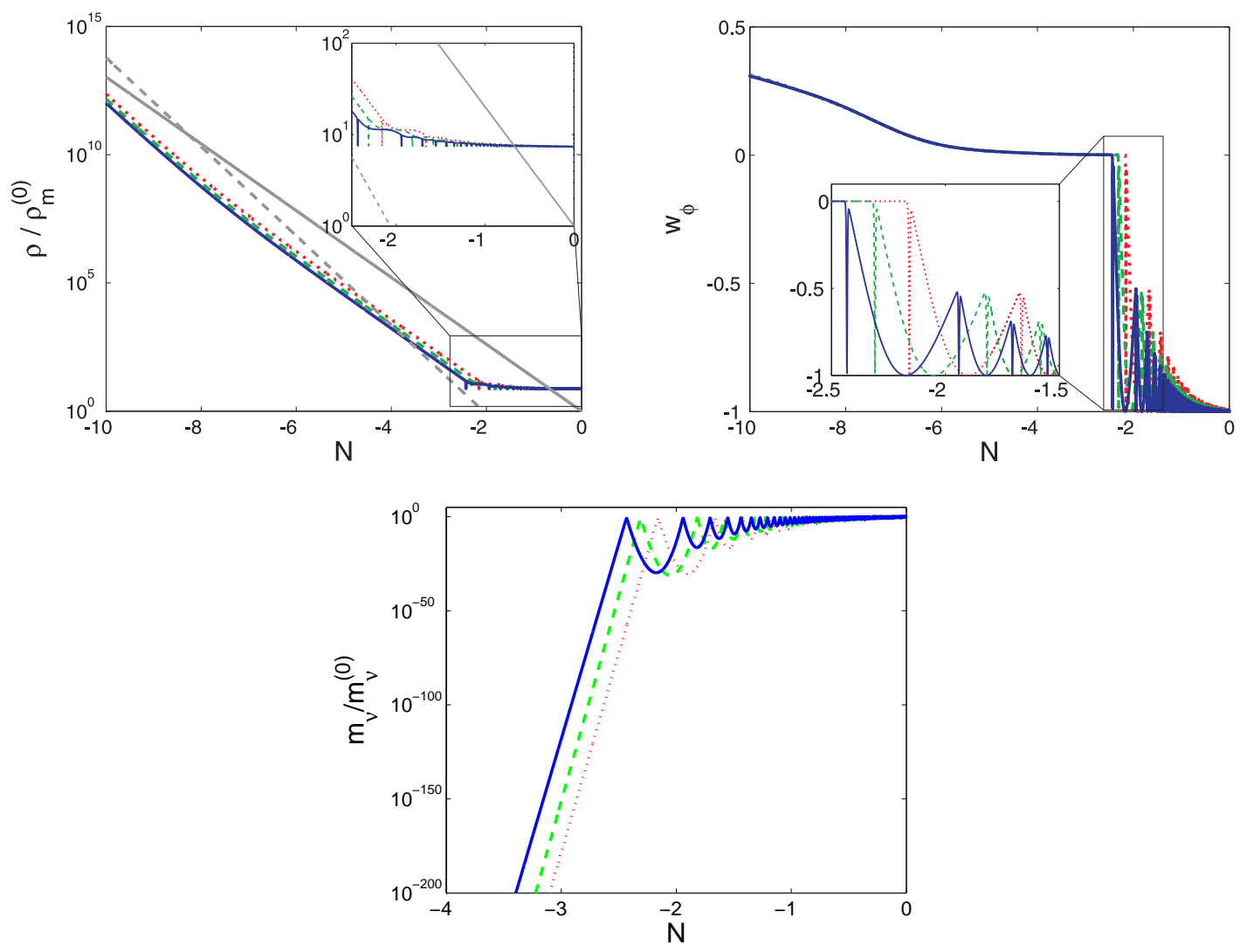

FIG. 3: Upper left: Evolutions of the various energy densities, normalized with the matter energy density $\rho_{m}^{(0)}$ at present $\left(a_{0}=1\right)$, as functions of $N \equiv \ln a$, for the non-minimally coupled scenario, with the effective potential $V_{e f f, \phi}=-\alpha V_{0} e^{-\alpha \phi / M_{P l}} / M_{P l}+\beta\left(\rho_{\nu}-3 p_{\nu}\right) / M_{P l}$, where $\rho_{r}$ is the graydashed curve, $\rho_{m}$ is the gray-solid curve, and $\rho_{\phi}$ is plotted for three parameter choices, namely $\alpha=30$ (blue-solid), 25 (green-dashed) and 20 (red-dotted), with $V_{0} / \rho_{m}^{(0)}=7 / 3$. Upper right: The corresponding evolution of the quintessence equation-of-state parameter $w_{\phi}$. Lower: Evolutions of the neutrino masses relative to the current masses. We have used $\Sigma m_{\nu}=0.2 \mathrm{eV}, \Omega_{m} h^{2}=0.118$ and $\rho_{r}^{(0)} / \rho_{m}^{(0)}=2.58 \times 10^{-4}$ as the boundary conditions.

which corresponds to the dark energy scale. For this $V_{0}$ choice, the final scalar approaches to the origin of coordinate, i.e., $\phi \sim 0$ at $z=0$. Furthermore, we should also mention that in this work we focus on the simplest varying neutrino-mass model, with only one neutrino flavor having the field varying mass, while the other two are treated as massless.

As the matter density perturbation is suppressed by the free-streaming massive neutrino, the neutrino mass influences the matter power spectrum. However, since small neutrino masses are preferred for non-minimally coupled neutrino matter in the early time (see also 
the lower panel in Fig.3), the suppression in the matter power spectrum due to massive neutrinos is minimized. In the upper panel of Fig. 4, we depict the matter power spectrum with $\Sigma m_{\nu}=0.04$ and $0.15 \mathrm{eV}$, where the measured data points are from the Sloan Digital Sky Survey (SDSS DR7). In the lower panel of Fig. 4, we plot the matter power spectrum deviation between $\Sigma m_{\nu}=0.15 \mathrm{eV}$ and $0.04 \mathrm{eV}$ in coupled neutrino matter (black solid line) and $\Lambda \mathrm{CDM}$ (gray solid line) models. Compared to $\Lambda \mathrm{CDM}$, we can see that in the nonminimally coupled neutrino matter model the suppression of the matter power spectrum is minimized due to the free-streaming neutrino.

As shown in Refs. [26, 27], the scalar $\phi$ within a large scale neutrino lump largely deviates from that at the background level $\bar{\phi}$, leading the neutrino mass to become negligible inside the neutrino lump, and thus the perturbation to become non-linear. In this work we desire to avoid such a non-linear region and we assume that the neutrino mass $m_{\nu}$ and the scalar $\phi$ are both homogeneous, and therefore the perturbation of neutrinos in the nonminimally-coupled scenario behaves as that in the $\Lambda$ CDM paradigm:

$$
\dot{\delta}_{\nu}=3 H\left(w_{\nu}-\frac{\delta p_{\nu}}{\delta \rho_{\nu}}\right) \delta_{\nu}-\left(1+w_{\nu}\right)\left(\theta-\frac{\dot{h}}{2}\right) .
$$

Finally, since in the scenario at hand the dark energy sector is attributed to the combination of the quintessence field and the varying-mass neutrinos according to (14) and (15), for completeness in Fig. 5 we depict the evolution of $w_{\phi}=p_{\phi} / \rho_{\phi}$ along with $w_{D E}=\left(p_{\phi}+p_{\nu}\right) /\left(\rho_{\phi}+\rho_{\nu}\right)$ and $w_{\nu}=p_{\nu} / \rho_{\nu}$. As expected according to our previous discussion, at late times $w_{\phi}$ determines $w_{D E}$ almost completely. In addition, from (20) we estimate that

$$
\beta \simeq \frac{\alpha V\left(\phi_{\min }\right)}{\rho_{\nu}} \simeq \frac{32 \alpha}{\Sigma m_{\nu} / \mathrm{eV}}
$$

where we have used $\rho_{\phi} \simeq V\left(\phi_{\text {min }}\right), \Omega_{\phi} h^{2}=0.34$ and $\Omega_{\nu} h^{2}=\Sigma m_{\nu} / 94.1 \mathrm{eV}$. Combined with (13), the small fluctuation of $\phi$ results in a significant change in neutrino masses. As shown in Fig. 5, neutrino masses along with the equation-of-state parameters oscillate in $-2 \lesssim \ln a \lesssim-0.5$, i.e. $0.7 \lesssim z \lesssim 6.4$, and become purely massive after $z \lesssim 0.7$.

As the scenario at hand leads to a successful description of the universe history at the background level, it is necessary to confront it with observations-related matter perturbations, as the varying-mass neutrino could in principle lead to strong constraints in this case. In the following section we perform such an observational analysis in detail. 

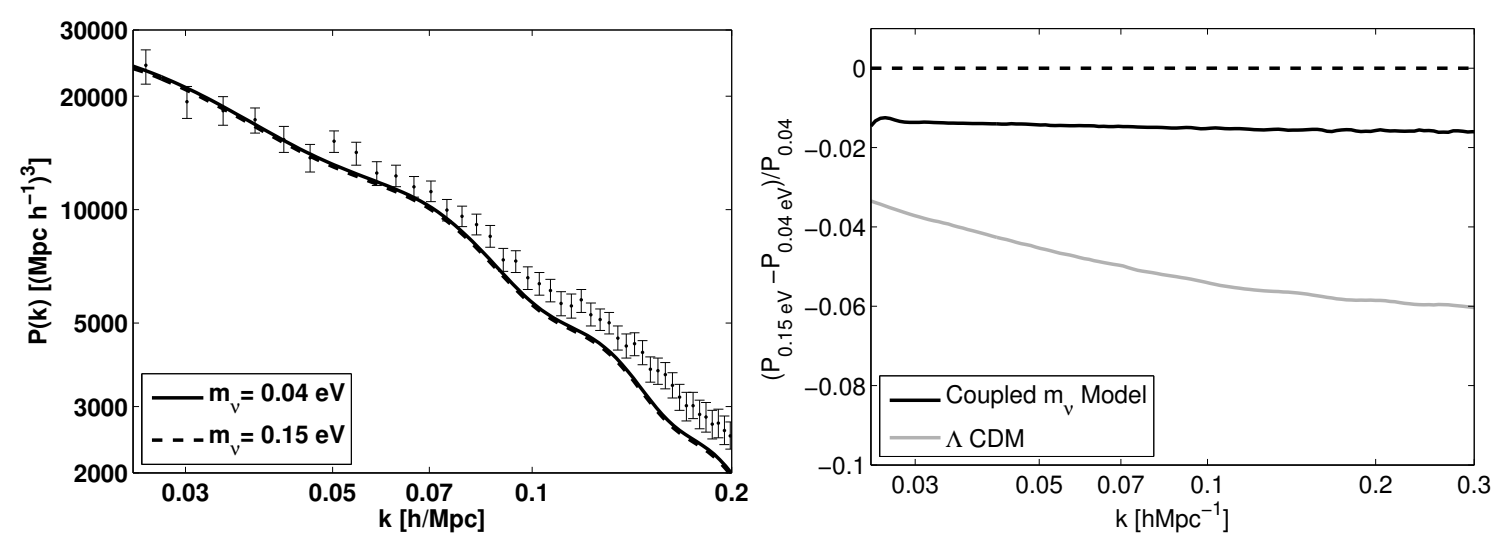

FIG. 4: Left: Matter power spectrum of the non-minimally coupled neutrino matter model with $V_{e f f, \phi}=-\alpha V_{0} e^{-\alpha \phi / M_{P l}} / M_{P l}+\beta\left(\rho_{\nu}-3 p_{\nu}\right) / M_{P l}$, where $\Sigma m_{\nu}=0.04 \mathrm{eV}$ (solid) and $0.15 \mathrm{eV}$ (dashed), $V_{0} / \rho_{m}^{(0)}=7 / 3$ as the boundary condition and $\left(\alpha, \Sigma m_{\nu} / \mathrm{eV}, \Omega_{c} h^{2}, 100 \Omega_{b} h^{2}\right)=(25,0.2,0.118,2.20)$. Right: the deviation of the matter power spectrum $\Delta P^{C} \equiv\left(P_{0.15}^{C}-P_{0.04}^{C}\right) / P_{0.04}^{C}$ in coupled neutrino matter model (black solid line) and $\Delta P^{\Lambda C D M} \equiv\left(P_{0.15}^{\Lambda C D M}-P_{0.04}^{\Lambda C D M}\right) / P_{0.04}^{\Lambda C D M}$ in $\Lambda C D M$ model (gray solid line), where $P_{0.15,0.04} \equiv P(k)$ with the neutrino mass $\Sigma m_{\nu}=0.15$ and $0.04 \mathrm{eV}$.

\section{OBSERVATIONAL CONSTRAINTS}

We use the program CosmoMC $[44,45]$ to extract the observational constraints on the scenario of the previous section. An important point is that one should go beyond the background evolution, since the massive neutrino effects will also arise from the matter density perturbation. Therefore, in our analysis we incorporate the matter powerspectrum data sets, which include cosmic microwave background (CMB) from Planck [9] and WMAP [47], baryon acoustic oscillation (BAO) from Baryon Oscillation Spectroscopic Survey (BOSS) [48, 49], Type-Ia supernova (SNIa) from Supernova Legacy Survey (SNLS) [50], and matter power spectrum from Sloan Digital Sky Survey (SDSS DR4) [51] and WiggleZ Dark Energy Survey [52, 53]. The details of the fitting procedure can be found in Refs. [44, 45]. In Fig. 6, we depict the 2D likelihood contours for the mass sum of the three active neutrinos at present $(z=0)$ and the model parameter $\alpha$, versus the physical density of cold dark matter $(\mathrm{CDM}) \Omega_{c} h^{2}$. The scenario at hand is in agreement with observations, and indeed quantities like the present dark-matter and dark-energy density parameters have similar ranges as those in $\Lambda \mathrm{CDM}$ cosmology [9]. However, the neutrino mass sum in the model is enhanced to $0.28 \mathrm{eV}$ and the allowed window is also significantly relaxed such that 


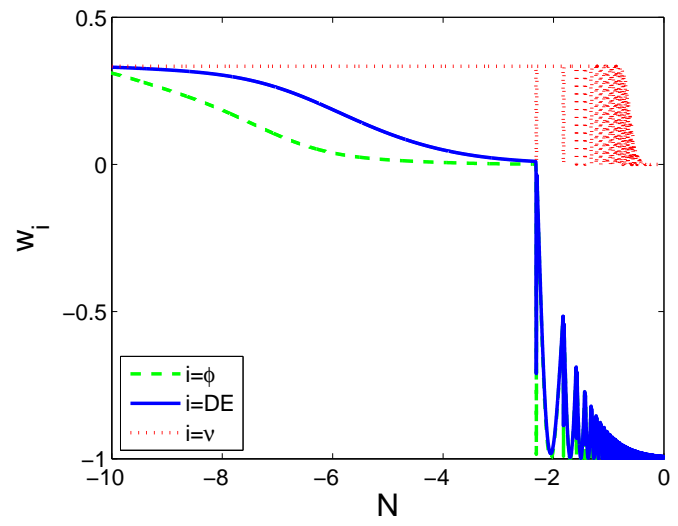

FIG. 5: Evolutions of the equation-of-state parameters of the total dark-energy sector $w_{D E}$ (blue-solid), of the quintessence field $w_{\phi}$ (green-dashed), and of the neutrinos $w_{\nu}$ (red-dotted), as functions of $N \equiv \ln a$, for the non-minimally coupled scenario, with the effective potential $V_{e f f, \phi}=-\alpha V_{0} e^{-\alpha \phi / M_{P l}} / M_{P l}+\beta\left(\rho_{\nu}-3 p_{\nu}\right) / M_{P l}$, where we have used $\alpha=25$ and $V_{0} / \rho_{m}^{(0)}=7 / 3$ as well as $\Sigma m_{\nu}=0.2 \mathrm{eV}, \Omega_{m} h^{2}=0.118$ and $\rho_{r}^{(0)} / \rho_{m}^{(0)}=2.58 \times 10^{-4}$ as the boundary conditions .
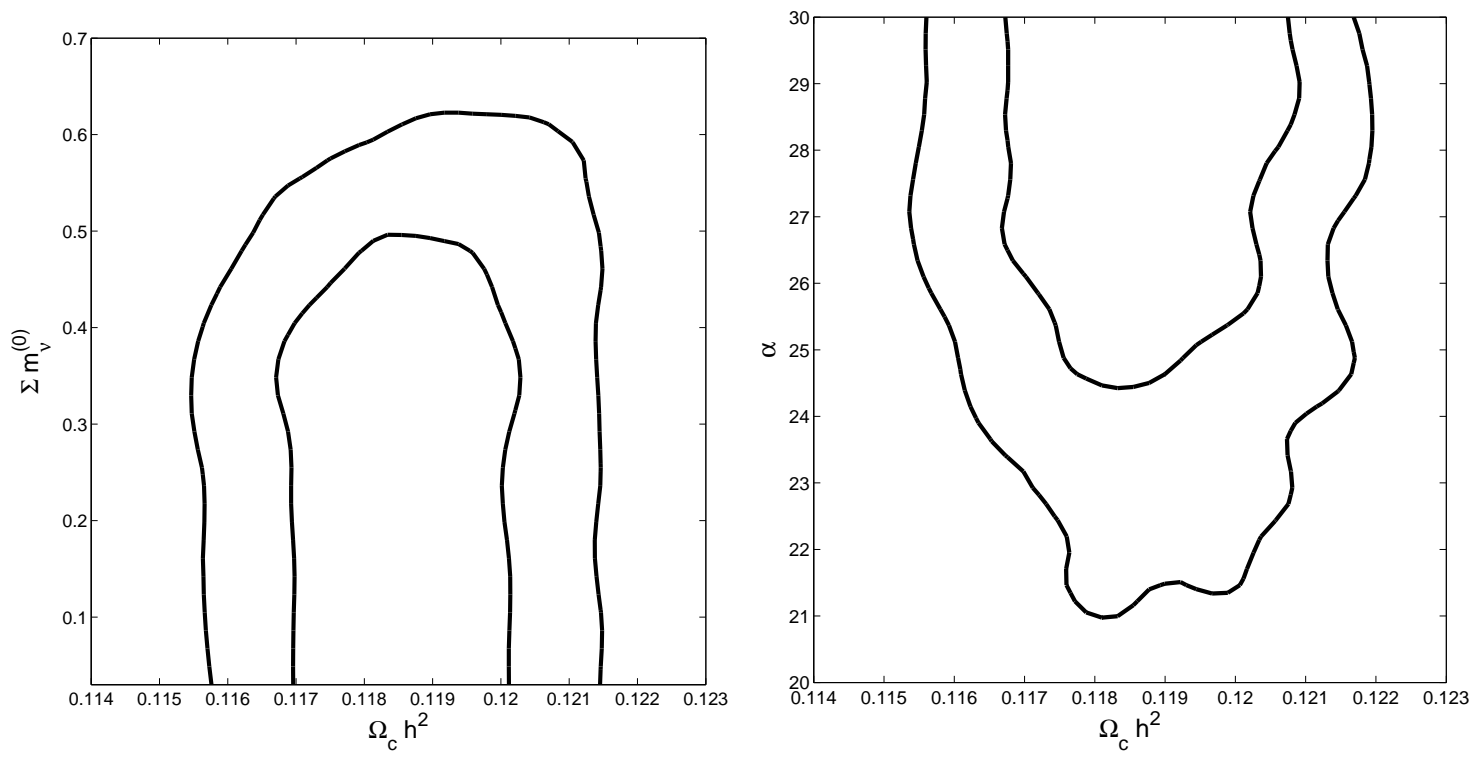

FIG. 6: Likelihood contours of the sum of the mass of the three neutrino species $\Sigma m_{\nu}^{z=0}$ in $e V$ (upper) and the potential parameter $\alpha$ (lower) versus the CDM physical density $\Omega_{c} h^{2}$, for the non-minimally coupled scenario with the effective potential $V_{e f f, \phi}=-\alpha V_{0} e^{-\alpha \phi / M_{P l}} / M_{P l}+\beta\left(\rho_{\nu}-\right.$ $\left.3 p_{\nu}\right) / M_{P l}$, where the inner and outer curves represent 1 and $2 \sigma$ confidence levels, respectively. 
TABLE II: List of priors for parameters and allowed region with 95\% C.L., and $V_{e f f, \phi}=$ $-\alpha V_{0} e^{-\alpha \phi / M_{\mathrm{Pl}}} / M_{\mathrm{Pl}}+\beta\left(\rho_{\nu}-3 p_{\nu}\right) / M_{\mathrm{Pl}}$.

\begin{tabular}{|l||c|c|c|}
\hline \hline Parameter & Prior & Our result $(95 \%$ C.L. $)$ & $\Lambda$ CDM $(95 \%$ C.L. $)$ \\
\hline Baryon density & $0.5<100 \Omega_{b} h^{2}<10$ & $100 \Omega_{b} h^{2}=2.19_{-0.05}^{+0.04}$ & $100 \Omega_{b} h^{2}=2.22_{-0.06}^{+0.04}$ \\
\hline CDM density & $10^{-3}<\Omega_{c} h^{2}<0.99$ & $\Omega_{c} h^{2}=0.119 \pm 0.003$ & $\Omega_{c} h^{2}=0.117_{-0.002}^{+0.004}$ \\
\hline Neutrino mass & $0.03<\Sigma m_{\nu}<1 \mathrm{eV}$ & $\Sigma m_{\nu}=0.278_{-0.248}^{+0.245} \mathrm{eV}$ & $\Sigma m_{\nu}<0.198 \mathrm{eV}$ \\
\hline Spectral index & $0.9<n_{s}<1.1$ & $n_{s}=0.963_{-0.011}^{+0.010}$ & $n_{s}=0.963_{-0.011}^{+0.010}$ \\
\hline Potential & $20<\alpha<30$ & $\alpha>22.1$ & - \\
\hline
\end{tabular}

$\Sigma m_{\nu}<0.52 \mathrm{eV}$. The potential parameter $\alpha$ controls the ratio $\rho_{\phi} / \rho_{c}$ in the high redshift regime, which at the $2-\sigma$ confidence level is constrained to be $\alpha>22.1$, which naturally yields the tracker behavior so that the coincident problem could be understood. Our results for the non-minimally coupled scenario are summarized in Table. II. Finally, it is worth to mention that the coupling $\beta$ is tuned to control $\Omega_{\phi}$ in our program, and its value is roughly inversely proportional to $\alpha$ and $\Sigma m_{\nu}$. In our numerical computation, we take $\beta \sim 800 / \Sigma m_{\nu}$ with $\alpha=25$. This can be estimated by the relation of Eq. (24).

\section{CONCLUSIONS}

We have examined the tracker quintessence models with inverse power-law and doubleexponential potentials. In the first case, it is difficult to obtain a viable scenario for generic values of model parameters, whereas in the second case we are forced to make a natural choice for the scale of the potential which brings back the problem that cosmological constant is plagued with. With a hope to alleviate the problem, we have considered massive neutrino matter non-minimally coupled to gravity. During radiation and early matter era, massive neutrinos exhibit relativistic behavior which implies vanishing coupling to scalar field. Only at late times, when massive neutrinos turn non-relativistic, their direct coupling to field builds up, leading to appearance of a minimum in the effective potential of the field. In this picture, the minimum of the effective potential is insensitive to the scale of the potential $V_{0}$ and is rather given by the numerical value of $\rho_{\nu}$ around the present epoch. At late times, when the scalar field rolls around the minimum of the effective potential, the dynamical 
system enters into scaling regime, which is an attractor. Thanks to non-minimal coupling, the scaling solution is accelerating. Clearly, the model under consideration has an edge over standard quintessence with flat potential.

We have found that the neutrino mass grows in time and its mass in the past is smaller than the current stage. In the early universe, the free-streaming length of the neutrino around the horizon scale is the same as that of the massless one. As shown in Fig. 5, the neutrinos become massive only at the very recent epoch. As a result, our scenario can relax the suppression of the matter power spectrum from the free-streaming massive neutrino in the early universe. The constraint on the neutrino masses is reduced in comparison to $\Lambda \mathrm{CDM}$. In particular, in the scenario under consideration, we found that the best-fit value of the neutrino mass sum are around $0.28 \mathrm{eV}$, along with the allowed window extended to $0.52 \mathrm{eV}$, which is consistent with particle physics experiments.

\section{Acknowledgments}

MS and ENS wish to thank National Center for Theoretical Sciences, Hsinchu, Taiwan for the hospitality during the initial stages of this work. This work was partially supported by National Center for Theoretical Sciences, National Science Council (NSC-101-2112-M007-006-MY3), MoST (MoST-104-2112-M-007-003-MY3) and National Tsing Hua University (104N2724E1).

[1] A. A. Starobinsky, Phys. Lett. B 91, 99 (1980).

[2] A. H. Guth, Phys. Rev. D 23, 347 (1981).

[3] A. D. Linde, Phys. Lett. B 108, 389 (1982).

[4] L. M. Krauss and M. S. Turner, Gen. Rel. Grav. 27, 1137 (1995) [astro-ph/9504003].

[5] A. G. Riess et al. [Supernova Search Team Collaboration], Astron. J. 116, 1009 (1998) [astro-ph/9805201].

[6] S. Perlmutter et al. [Supernova Cosmology Project Collaboration], Astrophys. J. 517, 565 (1999) [astro-ph/9812133].

[7] D. N. Spergel et al. [WMAP Collaboration], Astrophys. J. Suppl. 148, 175 (2003) [as- 
tro-ph/0302209].

[8] A. E. Lange et al. [Boomerang Collaboration], Phys. Rev. D 63, 042001 (2001) [astro-ph/0005004].

[9] P. A. R. Ade et al. [Planck Collaboration], Astron. Astrophys. (2014) [arXiv:1303.5076 [astroph.CO]].

[10] P. J. E. Peebles and B. Ratra, Rev. Mod. Phys. 75, 559 (2003) [astro-ph/0207347].

[11] Y. Fujii, Phys. Rev. D 26, 2580 (1982).

[12] L. H. Ford, Phys. Rev. D 35, 2339 (1987).

[13] B. Ratra and P. J. E. Peebles, Phys. Rev. D 37, 3406 (1988).

[14] C. Wetterich, Nucl. Phys. B 302, 645 (1988).

[15] G. t Hooft, Naturalness, Chiral Symmetry, and Spontaneous Chiral Symmetry Breaking, Recent Developments in Field Theories, ed. G.t Hooft et al., Plenum Press, New York, 1980.

[16] Y. Fujii and K. Maeda, The Scalar-Tensor Theory of Gravitation Cambridge Monographs on Mathematical Physics, Cambridge Univ. Press, 2003.

[17] V. Faraoni, Cosmology in Scalar-Tensor Gravity, Fundamental Theories of Physics, Springer, Heidelberg, 2004.

[18] P. J. E. Peebles and A. Vilenkin, Phys. Rev. D 60,103506 (1999) [astro-ph/9904396].

[19] E. J. Copeland, A. R. Liddle and J. E. Lidsey, Phys. Rev. D 64, 023509 (2001) [astro-ph/0006421.

[20] V. Sahni, M. Sami and T. Souradeep, Phys. Rev. D 65, 023518 (2001) [gr-qc/0105121].

[21] M. Sami and V. Sahni, Phys. Rev. D 70, 083513 (2004) [hep-th/0402086].

[22] M. W. Hossain, R. Myrzakulov, M. Sami and E. N. Saridakis, [1410.6100[gr-qc]].

[23] C. Wetterich, Phys. Rev. D 89, 024005 (2014) [1308.1019[astro-ph.CO]].

[24] M. W. Hossain, R. Myrzakulov, M. Sami and E. N. Saridakis, Phys. Rev. D 90, 023512 (2014) [1402.6661 [gr-qc]].

[25] M. W. Hossain, R. Myrzakulov, M. Sami and E. N. Saridakis, Phys. Rev. D 89, 123513 (2014) [1404.1445 [gr-qc]].

[26] D. F. Mota, V. Pettorino, G. Robbers and C. Wetterich, Phys. Lett. B 663 (2008) 160 doi:10.1016/j.physletb.2008.03.060 [arXiv:0802.1515 [astro-ph]].

[27] Y. Ayaita, M. Baldi, F. Fuhrer, E. Puchwein and C. Wetterich, arXiv:1407.8414 [astro-ph.CO].

[28] J. Lesgourgues and S. Pastor, "Massive neutrinos and cosmology," Phys. Rept. 429, 307 (2006) 
[astro-ph/0603494].

[29] C. Q. Geng, C. C. Lee and J. L. Shen, Phys. Lett. B 740, 285 (2015) [arXiv:1411.3813 [astroph.CO]].

[30] R. Fardon, A. E. Nelson and N. Weiner, JCAP 0410, 005 (2004) [astro-ph/0309800].

[31] R. D. Peccei, Phys. Rev. D 71, 023527 (2005) [hep-ph/0411137].

[32] A. W. Brookfield, C. van de Bruck, D. F. Mota and D. Tocchini-Valentini, Phys. Rev. D 73, 083515 (2006) [astro-ph/0512367].

[33] L. Amendola, M. Baldi and C. Wetterich, Phys. Rev. D 78, 023015 (2008) [arXiv:0706.3064 [astro-ph]].

[34] N. Wintergerst, V. Pettorino, D. F. Mota and C. Wetterich, Phys. Rev. D 81, 063525 (2010) [arXiv:0910.4985 [astro-ph.CO]].

[35] G. La Vacca and D. F. Mota, Astron. Astrophys. 560, A53 (2013) [1205.6059 [astro-ph.CO]].

[36] E. J. Copeland, M. Sami and S. Tsujikawa, Int. J. Mod. Phys. D 15, 1753 (2006) [hep-th/0603057].

[37] J. P. Kneller and L. E. Strigari, Phys. Rev. D 68, 083517 (2003) [astro-ph/0302167].

[38] L. R. W. Abramo and F. Finelli, Phys. Lett. B 575, 165 (2003) [astro-ph/0307208].

[39] E. N. Saridakis, Nucl. Phys. B 819, 116 (2009) [arXiv:0902.3978 [astro-ph.CO]].

[40] T. Barreiro, E. J. Copeland and N. J. Nunes, Phys. Rev. D 61, 127301 (2000) [astro-ph/9910214].

[41] T. Gonzalez, G. Leon and I. Quiros, Class. Quant. Grav. 23, 3165 (2006) [astro-ph/0702227].

[42] T. Gonzalez, R. Cardenas, I. Quiros and Y. Leyva, Astrophys. Space Sci. 310, 13 (2007) [arXiv::0707.2097 [astro-ph]].

[43] Bean R., Hansen S. H., Melchiorri A., 2002, Nucl. Phys. Proc. Suppl. 110167

[44] A. Lewis, A. Challinor and A. Lasenby, Astrophys. J. 538, 473 (2000) [astro-ph/9911177].

[45] A. Lewis and S. Bridle, Phys. Rev. D 66, 103511 (2002) [astro-ph/0205436].

[46] M. W. Hossain, R. Myrzakulov, M. Sami and E. N. Saridakis, Phys. Lett. B 737, 191 (2014) [arXiv:1405.7491 [gr-qc]].

[47] G. Hinshaw et al. [WMAP Collaboration], Astrophys. J. Suppl. 208, 19 (2013) [arXiv:1212.5226 [astro-ph.CO]].

[48] L. Anderson, E. Aubourg, S. Bailey, D. Bizyaev, M. Blanton, A. S. Bolton, J. Brinkmann and J. R. Brownstein et al., Mon. Not. Roy. Astron. Soc. 427, 3435 (2013) [arXiv:1203.6594 
[astro-ph.CO]].

[49] L. Anderson et al. [BOSS Collaboration], Mon. Not. Roy. Astron. Soc. 441, 24 (2014) [arXiv:1312.4877 [astro-ph.CO]].

[50] P. Astier et al. [SNLS Collaboration], Astron. Astrophys. 447, 31 (2006) [astro-ph/0510447].

[51] J. K. Adelman-McCarthy et al. [SDSS Collaboration], Astrophys. J. Suppl. 162, 38 (2006) [astro-ph/0507711].

[52] C. Blake, T. Davis, G. Poole, D. Parkinson, S. Brough, M. Colless, C. Contreras and W. Couch et al., Mon. Not. Roy. Astron. Soc. 415, 2892 (2011) [arXiv:1105.2862 [astro-ph.CO]].

[53] C. Blake, E. Kazin, F. Beutler, T. Davis, D. Parkinson, S. Brough, M. Colless and C. Contreras it et al., Mon. Not. Roy. Astron. Soc. 418, 1707 (2011) [arXiv:1108.2635 [astro-ph.CO]].

\section{APPENDIX: THE INVERSE POWER-LAW POTENTIAL}

For completeness, let us investigate the case of the power-law potential (5). In this case, the evolution equations can be explicitly integrated in the background (radiation/matter) dominated regime. The asymptotic behavior of the solution in the radiation and matter dominated eras is given by a power-law behavior with

$$
w_{\phi}=\frac{-2+n w_{b}}{n+2} .
$$

Eq. (25) implies that $w_{\phi}<w_{b}$ for $n>0$ in the radiation and matter dominated epochs, i.e.

$\rho_{\phi} / \rho_{m}^{(0)}$ increases, allowing finally to catch up with dark-energy domination. For moderate values of $n \sim 1$, the field might take over the background and as a result $\rho_{\phi}$ might obtain the observed value of dark energy at the present epoch, whereas $\omega_{\phi}$ has not yet reached the desired value. In the upper graph of Fig. 7, we depict the corresponding evolutions for the energy densities of radiation $\left(\rho_{r}\right)$, matter $\left(\rho_{m}\right)$ and quintessence field $\left(\rho_{\phi}\right)$, normalized with the matter energy density $\rho_{m}^{(0)}$ at present $\left(a_{0}=1\right)$, as functions of $N \equiv \ln a$, for the case $n=4$ with three choices of $V_{0}$. In the lower graph of Fig. 7 , we show the corresponding evolution for the quintessence equation-of-state parameter $w_{\phi}$. From this plot we can see that there exist two regimes with constant values of $w_{\phi} \approx-1 / 9$ and $w_{\phi} \approx-1 / 3$, in the radiation and matter dominated era, where the field tracks the background. Clearly, the equation-of-state parameter $w_{\phi}$ does not reach the required value at the present epoch.

The above situation can be remedied by taking large values of $n$, for which $w_{\phi} \rightarrow w_{b}$. In 

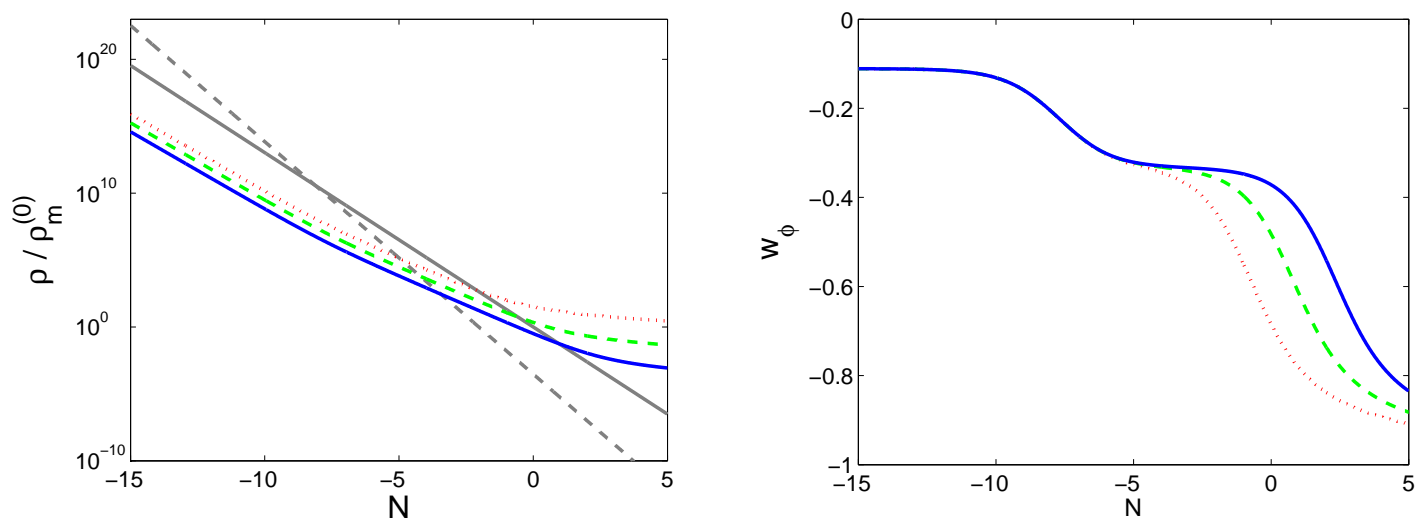

FIG. 7: Left: Evolutions of the various energy densities, normalized with the matter energy density $\rho_{m}^{(0)}$ at present $\left(a_{0}=1\right)$, as a function of $N \equiv \ln a$, for the minimally coupled scenario with the inverse power-law potential $V_{0}\left(\frac{\phi}{M_{P l}}\right)^{-n}: \rho_{r}$ is the gray-dashed curve, $\rho_{m}$ is the gray-solid curve, and $\rho_{\phi}$ is plotted for three parameter choices, namely $\left(n, V_{0} / \rho_{m}^{(0)}\right)=(4,1)$ (blue-solid), $\left(n, V_{0} / \rho_{m}^{(0)}\right)=$ $\left(4,10^{2}\right)$ (green-dashed) and $\left(n, V_{0} / \rho_{m}^{(0)}\right)=\left(4,10^{4}\right)$ (red-dotted). Right: The corresponding evolution of the quintessence equation-of-state parameter $w_{\phi}$. We have used $\rho_{r}^{(0)} / \rho_{m}^{(0)}=3 \times 10^{-4}$ as the boundary condition.

this case, the inverse power-law potentials reduce to the exponential form, leading to scaling solution. The late-time exit from the scaling regime is guaranteed by the shallow nature of inverse power-law functions, though we need to tune the model parameters appropriately. Firstly, irrespectively of the quintessence model, using the slow-roll parameter $\eta$ we find that $m_{\phi} \sim H_{0} \sim 10^{-33} \mathrm{eV}$. The slow-roll parameter $\epsilon$ tells us that this would happen at the present epoch, provided that $\phi_{0} / M_{\mathrm{Pl}}>>\sqrt{2} n$. Hence, this implies that $V_{0} \sim n^{n} \rho_{c r}$. In the case where $V_{0} \sim M_{p}$, one requires a large value of $n$. On the other hand, for moderate values of $n$ we need to choose $V_{0} \sim \rho_{c r}$ (Sahni, Sami \& Souradeep [20]). 